J. Klin. Endokrinol. Stoffw. $2020 \cdot 13: 177-181$ https://doi.org/10.1007/s41969-020-00124-7 Online publiziert: 6. November 2020 (c) Der/die Autor(en) 2020

\section{Gerlies Treiber}

Abteilung für Endokrinologie und Diabetologie, Universitätsklinik für Innere Medizin, Medizinische Universität Graz, Graz, Österreich

\title{
Nicht-Diabetes-assoziierte Hypoglykämien
}

In diesem Artikel erfolgt eine Übersicht über Ursachen von Hypoglykämien beim Erwachsenen ohne Diabeteserkrankung, deren klinischen Charakteristika sowie die empfohlenen Untersuchungen in der Abklärung. Dabei werden vor allem jene Erkrankungen dargestellt, welche zu einer endogenen Hyperinsulinämie führen. In der Differenzialdiagnose stellen die faktitiellen Hypoglykämien eine Herausforderung dar, auf diese wird hier nicht weiter eingegangen.

\section{Was passiert bei einer Hypoglykämie?}

Die Regulation des Blutzuckers im normoglykämischen Bereich erfolgt durch ein komplexes Regelwerk zwischen den Beta- und Alphazellen im endokrinen Teil des Pankreas, Glykogenolyse und Glukoneogenese in Leber sowie Muskel und Niere und dem Nervensystem mit unterschiedlicher sympathoadrenaler Aktivierung. Bei Abfall des Blutzuckers kommt es physiologischerweise zur Abnahme der Insulinsekretion, Steigerung der Glukagonausschüttung und in weiterer Folge zum Anstieg von Adrenalin, Cortison und Wachstumshormon mit dem Ziel, Glukose aus der endogenen Produktion zu mobilisieren [1].

Die häufigsten Ursachen für Hypoglykämien sind bekannterweise die Insulintherapie sowie die antidiabetische Therapie mit Sulfonylharnstoffen oder den kaum mehr eingesetzten Gliniden. - Tab. 1 gibt eine Übersicht von Ursachen bzw. Erkrankungen welche zu Nicht-Diabetes-assoziierten Hypoglykämien führen können.

\section{Fallpräsentation}

Ein 30-jähriger Mann wurde wegen gehäuften Auftretens von Wesensveränderungen im Rahmen eines gastrointestinalen Infekts stationär aufgenommen. Bis vor zwei Jahren war der Patient gesund, dann bemerkte er Episoden mit Taubwerden des Munds, Schwindel, verschwommenem Sehen und Störungen in der Konzentration mit „Blackouts“. Angehörige berichten von kindhaftem Verhalten sowie unsinnigem Reden während dieser Phasen. Sechs Monate vor Aufnahme nahmen diese Episoden $z u$, und die Symptome traten nachts, vor Mahlzeiten auf und besserten sich durch Zufuhr von Kohlenhydraten. Er hat $7 \mathrm{~kg}$ in dieser Zeit zugenommen. Eine neurologische Abklärung erbrachte die Diagnose von Panikattacken. Er nahm keine Drogen oder Anabolika ein, jedoch wurden Eiweißpräparate im Rahmen seines Bodybuilder-Trainings eingenommen. In der Familie gab es keine Erkrankungen des Pankreas oder des Immunsystems sowie keine Einnahme von Diabetesmedikamenten. Bei Aufnahme hatte der Patient einen Blutzucker von $27 \mathrm{mg} / \mathrm{dl}$, und sein Gewicht lag bei $104 \mathrm{~kg}\left(B M I 32,5 \mathrm{~kg} / \mathrm{m}^{2}\right)$.

\section{Was ist die Whipple-Trias?}

Die Whipple-Trias besteht aus den klinischen adrenergen und/oder neuroglykopenischen Symptomen der Hypoglykämie (『Tab. 2) plus dokumentierter Blutzuckermessung mit Plasmaglukose $\leq 55 \mathrm{mg} / \mathrm{dl}$ (früher $\leq 45 \mathrm{mg} / \mathrm{dl}$ ) und einer raschen Besserung der Symptome durch Zufuhr von schnellresorbierbaren Kohlenhydraten. Die ausführliche Erhebung der Symptome, Zeitpunkt des Auftretens (nüchtern, nachts, postprandial),
Begleitumstände, Ernährungsgewohnheiten, Vorerkrankungen, Einnahme von Medikamenten, Nahrungsergänzungsmittel, Familienanamnese und soziale Anamnese sind wegweisend in der Abklärung von Nicht-Diabetes-assoziierten Hypoglykämien. Bei Vorliegen der Whipple-Trias erhebt sich der Verdacht einer endogenen Hyperinsulinämie, und weitere diagnostische Evaluierung ist angezeigt [2].

\section{Wie wird ein 72-Stunden- Fastentest durchgeführt?}

Der 72-h-Fastentest ist weiter der Goldstandard in der Diagnose von autonomen endogenen Hyperinsulinämien. Bis zu $90 \%$ der Patienten mit Insulinomen bekommen Hypoglykämien in den ersten $48 \mathrm{~h}$ des Fastentests. Dabei ist die standardisierte Durchführung mit sorgfältiger Erhebung von Symptomen und Dokumentation der Blutabnahmen für die Interpretation essenziell. Im stationären Setting bekommt der Patient kein Essen für bis zu $72 \mathrm{~h}$, aber er darf Wasser trinken. Die parallele Blutabnahme für Glukose, Insulin, C-Peptid, Proinsulin und Ketone sollen alle $6 \mathrm{~h}$ durchgeführt werden. Wenn der Blutzucker $(\mathrm{BZ})<60 \mathrm{mg} / \mathrm{dl}$ sinkt, dann erfolgen die Blutabnahmen stündlich bzw. wenn eindeutig neuroglykopenische Symptome einer Hypoglykämie auftreten. Für einen positiven Fastentest muss eine dokumentierte Hypoglykämie (Serumglukose $<55 \mathrm{mg} / \mathrm{dl}$ ) vorliegen. Am Ende des Fastentests wird $1 \mathrm{mg}$ Glukagon intravenös gegeben und die Plasmaglukose nach 10, 20 und $30 \mathrm{~min}$ gemessen ([2]; - Tab. 3). 


\begin{tabular}{ll}
\hline Tab. 1 Ursachen von Hypoglykämien bei Erwachsenen ohne Diabeteserkrankung \\
$\begin{array}{ll}\text { Patient - "gesund“ } \\
\text { Endogene Hyperinsulinämie }\end{array}$ & Patient - „krank“ \\
\hline $\begin{array}{l}\text { Insulinom } \\
\text { Adulte Nesidioblastose (NIPHS) }\end{array}$ & $\begin{array}{l}\text { Endokrine Krisen (Addison) } \\
\text { Inanition }\end{array}$ \\
$\begin{array}{l}\text { Postbariatrische Hypoglykämien } \\
\text { Autoimmunes Insulinsyndrom }\end{array}$ & Leberversagen \\
Faktitielle Hypoglykämien (Insulin, SH, Glinide) & Nierenversagen \\
„Accidental, surreptitious, or malicious“ & Sepsis \\
& Noninslet-Cell-Tumoren \\
& Medikamente (z. B. Hydroxychloroquin, \\
& Lithium ...) \\
& Alkohol \\
\hline SH Sulfonylharnstoffe & \\
\hline
\end{tabular}

Tab. 2 Symptome Hypoglykämie

\begin{tabular}{|c|c|}
\hline Sympathoadrenerg & Neuroglykopenisch \\
\hline$<60-65 \mathrm{mg} / \mathrm{dl}$ & $<50-55 \mathrm{mg} / \mathrm{dl}$ \\
\hline Schwitzen & Verwirrung \\
\hline Schwäche & Reizbarkeit \\
\hline Hunger & Müdigkeit \\
\hline Herzrasen & Verändertes Verhalten \\
\hline Palpitationen & Sehstörungen \\
\hline Zittern & Neurologische Ausfälle \\
\hline Kribbeln & Krampfanfall \\
\hline Nervosität & Bewusstlosigkeit \\
\hline
\end{tabular}

Tab. 3 Ergebnisse des 72-h-Fastentests nach Ursache der Hypoglykämien und Werte des Patientenfalls

\begin{tabular}{|c|c|c|c|c|c|}
\hline & Patientenfall & Insulinom & Normal & Exogen Insulin & OAD \\
\hline Symptome & Ja & Ja & Nein & $\mathrm{Ja}$ & $\mathrm{Ja}$ \\
\hline Glukose & 28 & $<55 \mathrm{mg} / \mathrm{dl}$ & $\downarrow$ & $\downarrow \downarrow$ & $\downarrow \downarrow$ \\
\hline Insulin & 7,0 & $\geq 3 \mu \mathrm{IU} / \mathrm{ml}$ & $\downarrow$ & $\uparrow$ & $\uparrow$ \\
\hline C-Peptide & 2,2 & $\geq 0,6 \mathrm{ng} / \mathrm{ml}$ & $\downarrow$ & $\downarrow$ & $\uparrow$ \\
\hline Proinsulin & $>134$ & $\geq 5 \mathrm{pmol} / \mathrm{L}$ & $\downarrow$ & $\downarrow$ & $\uparrow$ \\
\hline Ketone & 0,02 & $\leq 2,7 \mathrm{mmol} / \mathrm{l}$ & $\uparrow \uparrow \uparrow$ & $\downarrow$ & $\downarrow$ \\
\hline $\begin{array}{l}\Delta \text { Glukose nach } \\
\text { Glukagon }\end{array}$ & $N d$ & $\geq 25 \mathrm{mg} / \mathrm{dl}$ & $<25 \mathrm{mg} / \mathrm{dl}$ & $\geq 25 \mathrm{mg} / \mathrm{dl}$ & $\geq 25 \mathrm{mg} / \mathrm{dl}$ \\
\hline Screen SH, Glinide & $N d$ & Negativ & Negativ & Negativ & Positiv \\
\hline
\end{tabular}

\section{Warum ist die parallele Messung von Insulin und C-Peptid sowie Proinsulin und Ketonkörpern ( $\beta$-Hydroxybutyrat) notwendig?}

Wenn C-Peptid mit Insulin erhöht ist, spricht dies für eine endogene Hyperinsulinämie. Wenn C-Peptid niedrig ist und Insulin erhöht, dann ist dies verdächtig auf eine exogene Insulinverabreichung im Rahmen einer Hypoglykämie factitia.

Proinsulin, eine Vorstufe des Insulins, wird nur $\mathrm{zu} 10 \%$ ins Blut abgegeben

\section{Weitere Abklärung bei positivem 72-h-Fastentest und Verdacht auf Insulinom?}

Diese funktionellen neuroendokrinen Tumoren sind selten (4/1 Mio. Patientenjahre), machen 1-2\% aller Pankreastumoren aus und führen zu Hypoglykämien durch endogene Hyperinsulinämie. Ein gehäuftes Auftreten liegt bei MEN 1 vor [4]. Zu $90 \%$ sind Insulinome gutartig und solitär und befinden sich im Pankreas. Die Lokalisationsdiagnostik kann sich aufgrund der Lage und der kleinen Größe schwierig gestalten. Routinemäßig werden eine Abdomensonografie, Computertomografie und/ oder Magnetresonanztomografie des Pankreas durchgeführt. Ein EUS (endoskopischer Ultraschall) hat eine höhere Sensitivität, kleinere Tumore zu finden. Somatostatin-Rezeptor-Szintigrafien mit ${ }^{68}$ Gallium-DOTANOC bzw. in manchen Fällen mit radioaktiv markiertem DOPA zeigen einen vermehrten Tracer-Uptake im Tumorgewebe. Eine weitere sehr hilfreiche Methode ist der SACT (Selektiver Kalzium-Stimulationstest) in der Lokalisation von Insulinomen, da Kalzium die Insulinausschüttung in den Insulinomzellen stimuliert [5-7].

\section{Patientenfall Lokalisations- diagnostik}

Bei unserem Patientenfall wurden folgende Untersuchungen durchgeführt: Abdomensonografie (kein Tumor), MR Pankreas (kein Tumor), ${ }^{68}$ Gallium-DOTANOCPET/CT-Szintigrafie (Tracer-Uptake in Cauda), DOPA-PET/CT-Szintigrafie (kein Tracer-Uptake), EUS (Tumor im Caput und Cauda, Zeichen chronischer Pankreatitis). Im SACT zeigte sich eine vermehrte Insulinausschüttung nach Kalziumgabe in der A. mesenterica superior (-Abb. 1). Ein weiteres PET/CT mit 68Ga-DOTAExendin-4, durchgeführt in Basel, zeigte den Nachweis einer $16 \times 8 \mathrm{~mm}$ messenden Raumforderung im Pankreaskopf rechts lateral der $V$. mesenterica superior. Es erfolgte eine Enukleation dieses Tumors, histologisch wurde ein Insulinom verifiziert, und der Patient war danach frei von Hypoglykämien. 
Die kausale Therapie des Insulinoms ist primär eine chirurgische Entfernung, wenn möglich Enukleation dieser Tumoren. Ist eine operative Sanierung nicht möglich, besteht die Möglichkeit einer Radiofrequenzablation oder medikamentöser Hemmung der Insulinsekretion mit Diazoxid, weiters können Somatostatinanaloga bei manchen Patienten effektiv sein. Bei malignen Insulinomen werden Everolimus, Sunitinib und Peptid-Radio-Rezeptor-Therapie (PRRT) eingesetzt $[8,9]$.

\section{Was ist eine Nesidioblastose?}

Klinisch wird die Nesidioblastose als NIPHS („non-insulinoma pancreatogenous hypoglycemia syndrome") bezeichnet und ist charakterisiert durch eine Hypertrophie der Betazellen. Diese führt vor allem zu postprandialen Hypoglykämien (•Tab. 4), während die Insulinome vorwiegend nüchtern $\mathrm{Hy}$ poglykämien verursachen. Roux-en-Ybariatrische Operationen können auch $\mathrm{zu}$ einer Nesidioblastose führen. Um diese Diagnose stellen zu können, wird neben dem negativen Fastentest ein Mahlzeitentoleranztest durchgeführt, wo sich Hypoglykämien induzieren lassen. In der radiologischen Bildgebung lässt sich kein Tumor darstellen, und im SACT zeigt sich eine vermehrte Insulinausschüttung aus multiplen Gefäßen $[10,11]$.

\section{Wann wird ein Mahlzeiten- toleranztest durchgeführt?}

Zeigen sich in der Anamnese vorwiegend postprandiale Hypoglykämiesymptome, wird ein Mahlzeitentoleranztest durchgeführt. Nach der basalen (nüchtern) Blutabnahme wird eine Mahlzeit konsumiert mit schnell resorbierbaren Kohlenhydraten. Bei Patienten mit bariatrischen Operationen soll keine Flüssignahrung dafür verwendet werden. Leider hat sich keine standardisierte Mahlzeit bisher etabliert (Kohlenhydratmenge variiert zwischen 40 und $75 \mathrm{~g}$ ). Blutabnahmen für Glukose, Insulin und C-Peptid werden alle $30 \mathrm{~min}$ bzw. stündlich für $5 \mathrm{~h}$ durchgeführt. Ergänzend werden noch Proinsulin und Insulin-Antikörper

J. Klin. Endokrinol. Stoffw. 2020 • 13:177-181 https://doi.org/10.1007/s41969-020-00124-7

(c) Der/die Autor(en) 2020

\section{G. Treiber}

\section{Nicht-Diabetes-assoziierte Hypoglykämien}

\section{Zusammenfassung}

Die Symptome einer Hypoglykämie sind häufig und unspezifisch. Echte Hypoglykämien sind selten und kommen meistens im Rahmen einer Insulintherapie vor. Die Diagnose einer Hypoglykämie bei Patienten ohne Diabetes erfordert das Vorliegen der Whipple-Trias mit dokumentierter Blutglukose $\leq 55 \mathrm{mg} / \mathrm{dl}$, hypoglykämischen Symptomen und deren Verschwinden durch Zufuhr von Kohlenhydraten. Eine weitere Abklärung erfolgt mittels standardisiertem 72-h Fastentest oder bei Patienten mit vorwiegend postprandialen Hypoglykämien einem 5-h-Mahlzeitentoleranztest. Die parallele Messung von Plasmaglukose, Insulin, C-Peptid, Proinsulin und Ketonen hilft in der Differenzierung zwischen Insulinom, faktitieller Gabe von Insulin und Sulfonylharnstoffen sowie Nesidioblastose (NIPHS) und Hypoglykämien nach bariatrischen Operationen. Die Messung von Insulin-Autoantikörpern ist hinweisend auf ein autoimmunes Insulinsyndrom und der Nachweis von „big“ IFG-2 auf eine paraneoplastische Sekretion dieses Proteins bei Tumorerkrankungen. Erst wenn der Nachweis einer autonomen endogenen Hyperinsulinämie vorliegt, erfolgt die Lokalisationsdiagnostik. Dabei sind szintigrafische Untersuchungen mittels radioaktivem ${ }^{68}$ Gallium und Exendin-4 und ein selektiver Kalzium-Stimulationstest in der Lokalisation von Insulinomen hilfreich. Therapeutisch ist eine Hypoglykämie akut durch Glukosegabe zu behandeln. Die langfristige Therapie besteht wenn möglich in der Beseitigung der auslösenden Ursache, Ernährungsmaßnahmen sowie medikamentöser Hemmung der Insulinsekretion.

\section{Schlüsselwörter}

Spontane Hypoglykämien · Whipple-

Trias · Hyperinsulinämie $\cdot 72$-h-Fastentest . Insulinom

\section{Hypoglycemia without diabetes}

\section{Abstract}

Symptoms of hypoglycemia are common and non-specific. Real hypoglycemia without insulin therapy is rare. The diagnosis of hypoglycemia in patients without diabetes requires the presence of the Whipple triad with documented blood glucose $\leq 55 \mathrm{mg} / \mathrm{dl}$, hypoglycemic symptoms and their relief after intake of glucose. A further diagnostic strategy is to replicate conditions in which hypoglycemia would be expected if a hypoglycemic disorder exists. A prolonged supervised fast, which can last as long as $72 \mathrm{~h}$, has been the best established and probably most reliable test for the evaluation of hypoglycemia occurring in the fooddeprived state. For patients with postprandial hypoglycemia, a mixed-meal test is the preferred provocative procedure. The parallel measurement of plasma glucose, insulin, C-peptide, proinsulin, and ketones helps in the differentiation between insulinoma, factitious administration of insulin and sulfonylureas as well as nesidioblastosis
(NIPHS) and hypoglycemia after bariatric surgery. Marked elevation of insulin autoantibodies is indicative for an autoimmune insulin syndrome and the detection of "big" IFG-2 relates to paraneoplastic secretion of this protein in tumor diseases. Localization diagnostics are only carried out when there is evidence of autonomic endogenous hyperinsulinemia. Scintigraphic examinations using ${ }^{68} \mathrm{Ga}$-labeled exendin-4, an interventional radiological technique, and the selective calcium stimulation test (SACT), can be performed to help the localization of insulinomas. Acute hypoglycemia must be treated by administering glucose. Long-term therapy consists, if possible, of eliminating the triggering cause, nutritional measures, and drug-based inhibition of insulin secretion.

\section{Keywords}

Spontaneous hypoglycemia $\cdot$ Hyperinsulinemia - Whipple triad $\cdot 72$-h fast . Insulinoma 


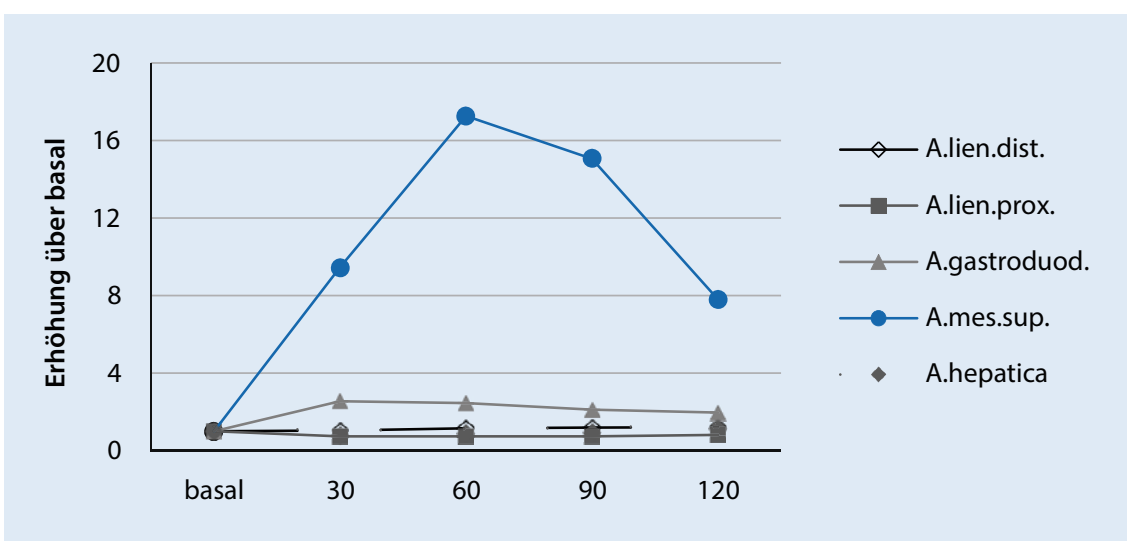

Abb. $1 \Delta$ Insulinkonzentrationen in der Vena hepatica zum Zeitpunkt 0 und 30, 60, 90 und 120 Sekunden nach arterieller Injektion von Kalziumglukonat während eines selektiven arteriellen Kalziumstimulationstests (SACT). Hier zeigt sich ein 17-facher Anstieg der Insulinkonzentration im Gebiet der A. mesenterica superior (Versorgung der kaudalen Anteilen des Caput pancreatis). A. lien. dist. Arteria lienalis distalis; . lien. prox. Arteria lienalis proximalis; A. gastroduod. Arteria gastroduodenalis; $A$. mes. sup. Arteria mesenterica superior

\begin{tabular}{l} 
Tab. 4 Ursachen für Auftreten von post- \\
prandialen Hypoglykämien \\
Postprandiale Hypoglykämien \\
\hline Nach bariatrischen Operationen \\
Nesidioblastose (NIPHS) \\
Autoimmunes Insulin-Syndrom \\
Insulinom (bis $6 \%$ nur postprandiale Hypo- \\
glykämien) \\
Gin Tonic Hypoglykämien \\
Ackee-Frucht-Vergiftung \\
Hereditäre Fruktoseintoleranz \\
Faktitielle Hypoglykämien
\end{tabular}

bestimmt. Ein verlängerter OGTT (oraler Glukosetoleranztest) wird in der Evaluation von postprandialen Hypoglykämien nicht mehr eingesetzt, da dabei auch bei Gesunden bis zu 10\% Hypoglykämien induziert werden [2].

\section{Warum kann es zu Hypo- glykämien nach bariatrischer Chirurgie kommen?}

Mit einer 5-Jahres-Inzidenz von $13 \%$ kommt es nach bariatrischen Eingriffen zu postprandialen Hypoglykämien, welche bei $1 \%$ auch $\mathrm{zu}$ bedrohlichen Symptomen mit Bewusstseinsstörungen und Krampfanfällen führen. Die Ursachen sind multifaktoriell und noch nicht gänzlich erforscht. Einerseits führen Inkretine wie GLP-1 (Glukagon-likePeptide) und GIP (Gastric-inhibitoryPeptide) zu einer gesteigerten postpran- dialen Insulinsekretion, die Gegenregulation im Rahmen der Hypoglykämie ist postoperativ reduziert; ferner kommt es durch die geänderte gastrointestinale Anatomie zu erhöhten zirkulierenden Gallensäuren. Eine Inselzellhyperplasie (Nesidioblastose) kann in seltenen Fällen auftreten. Die Behandlung dieser Hypoglykämien liegt in der intensiven diätologischen Betreuung mit Meiden von schnellresorbierbaren Kohlenhydraten. Medikamentös können Acarbose, Diazoxid oder Somatostatinanaloga eingesetzt werden $[12,13]$.

\section{Warum werden Insulin- Antikörper gemessen?}

Erhöhte Insulin-Antikörper sowie ausgeprägte Insulinspiegel (>1000 pmol/l) sind hinweisend auf ein autoimmunes Insulinsyndrom (Hirata-Krankheit). Patienten mit Autoimmunerkrankungen haben eine Prädisposition, und bestimmte Medikamente oder Virusinfektionen können Triggerfaktoren für die Ausbildung der Insulin-Antikörper sein. Sehr selten kommt es im Rahmen der Flier-Erkrankung (Typ-B-Insulinresistenz-Syndrom) zur Ausbildung von Antikörpern gegen den Insulinrezeptor. Bei diesem Krankheitsbild kommt es zu Nüchternhypoglykämien und auch insulinresistenten Hyperglykämien mit auch massiv erhöhten Insulinspiegeln und gleichzeitig reduzierten C-Peptidund Proinsulinwerten [14, 15].

\section{Wann ist die Messung von „big“ IGF-2 (Insulin-like Growth Factor 2) indiziert?}

Im Rahmen von Tumorerkrankungen (Non-Islet-Tumoren von mesenchymalem oder epithelialem Ursprung) kann es sehr selten paraneoplastisch zur Ausbildung von „big“ IFG2 kommen, einem Protein, welches den Insulinrezeptor stimuliert und vermehrt an IGF-Rezeptor bindet und somit die endogene Glukoseregulation stört und $\mathrm{zu}$ vor allem postprandialen Hypoglykämien führt. Laborchemisch sind eine Hypoinsulinämie, erniedrigte C-Peptid-Werte sowie reduziertes IGF1 und IFG-BR und erhöhtes „big“ IGF2 hinweisend [16].

\section{Fazit für die Praxis}

- Hypoglykämien mit Whipple-Trias sollen weiter abgeklärt werden.

- Goldstandard ist der 72-h-Fastentest mit gleichzeitiger Messung von Glukose, Insulin, C-Peptid, Proinsulin und Ketonen.

- Lokalisationsdiagnostik erst bei Nachweis einer autonomen endogenen Hyperinsulinämie.

- Das Insulinom ist ein seltener neuroendokriner Tumor, welcher vorwiegend nüchtern Hypoglykämien verursacht.

- Hypoglykämien nach bariatrischen Operationen treten typischerweise postprandial auf.

\section{Korrespondenzadresse}

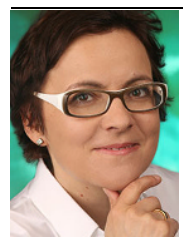

PD Dr. Gerlies Treiber

Abteilung für Endokrinologie und Diabetologie,

Universitätsklinik für Innere Medizin, Medizinische

Universität Graz Auenbruggerplatz 15, $8036 \mathrm{Graz}$, Österreich gerlies.treiber@medunigraz.at

Funding. Open access funding provided by Medical University of Graz. 


\section{Einhaltung ethischer Richtlinien}

Interessenkonflikt. G. Treiber gibt an, dass kein Interessenkonflikt besteht.

Für diesen Beitrag wurden von den Autoren keine Studien an Menschen oder Tieren durchgeführt. Für die aufgeführten Studien gelten die jeweils dort angegebenen ethischen Richtlinien.

Open Access. Dieser Artikel wird unter der Creative Commons Namensnennung 4.0 International Lizenz veröffentlicht, welche die Nutzung, Vervielfältigung, Bearbeitung, Verbreitung und Wiedergabe in jeglichem Medium und Format erlaubt, sofern Sie den/die ursprünglichen Autor(en) und die Quelle ordnungsgemäß nennen, einen Link zur Creative Commons Lizenz beifügen und angeben, ob Änderungen vorgenommen wurden.

Die in diesem Artikel enthaltenen Bilder und sonstige Drittmaterial unterliegen ebenfalls der genannten Creative Commons Lizenz, sofern sich aus der Abbildungslegende nichts anderes ergibt. Sofern das betreffende Material nicht unter der genannten Creative Commons Lizenz steht und die betreffende Handlung nicht nach gesetzlichen Vorschriften erlaubt ist, ist für die oben aufgeführten Weiterverwendungen des Materials die Einwilligung des jeweiligen Rechteinhabers einzuholen.

Weitere Details zur Lizenz entnehmen Sie bitte der Lizenzinformation auf http://creativecommons.org/ licenses/by/4.0/deed.de.

\section{Literatur}

1. Mitrakou A, Ryan C, Veneman T, Mokan M, Jenssen T, Kiss I, Durrant J, Cryer P, Gerich J (1991) Hierarchy of glycemic thresholds for counterregulatory hormone secretion, symptoms, and cerebral dysfunction. Am JPhysiol 260:E67-E74

2. Kittah E, Vella A (2017) Pathogenesis and management of hypoglycemia. Eur J Endocrinol 177:R37-R47. https://doi.org/10.1530/EJE-161062

3. Murtha TD (2017) A systematic review of proinsulin-secreting pancreatic neuroendocrine tumors. J Gastrointest Surg 21:1335-1341. https:// doi.org/10.1007/s11605-017-3428-8

4. Marx SJ (2018) Recent topics around multiple endocrine neoplasia type 1. J Clin Endocrinol Metab 103(4):1296-1301. https://doi.org/10. 1210/jc.2017-02340

5. Wang H, Ba Y, Xing Q, Cai RC (2019) Diagnostic value of ASVS for insulinoma localization: a systematic review and meta-analysis. PLoS ONE 14(11):e224928. https://doi.org/10.1371/journal. pone. 0224928

6. Christ E, Antwi K, Fani M, Wild D (2020) Innovative imaging of insulinoma: the end of sampling? A review. Endocr Relat Cancer. https://doi.org/10. 1530/erc-19-0476

7. Masciocchi M (2017) Pancreatic Imaging. Endocrinol Metab Clin North Am 46(3):761-781. https:// doi.org/10.1016/j.ecl.2017.04.006

8. Hofland J, Kaltsas G, de Herder WW (2019) Advances in the diagnosis and management of well-differentiated neuroendocrine neoplasms. Endocr Rev. https://doi.org/10.1210/endrev/ bnz004
9. Oberg K (2018) Management of functional neuroendocrine tumors of the pancreas. Gland Surg 7(1):20-27. https://doi.org/10.21037/gs. 2017.10.08

10. Service FJ, Natt N, Thompson GB, Grant CS et al (1999) Noninsulinoma pancreatogenous hypoglycemia: a novel syndrome of hyperinsulinemic hypoglycemia in adults independent of mutations in Kir6.2 and SUR1 genes. J Clin Endocrinol Metab 84:1582-1589. https://doi.org/10.1210/jc.84.5. 1582

11. Service FJ, Thompson GB, Service FJ et al (2005) Hyperinsulinemic hypoglycemia with nesidioblastosis after gastric-bypass surgery. N Engl J Med 353:249-254. https://doi.org/10 1056/NEJMoa043690

12. Salehi M, Vella A et al (2018) Hypoglycemia after gastric bypass surgery: current concepts and controversies. J Clin Endocrinol Metab 103:2815-2826. https://doi.org/10.1210/jc.201800528

13. Shah M, Law JH, Micheletto F, Sathananthan M, Dalla MC, Cobelli C, Rizza RA, Camilleri M Zinsmeister AR, Vella A (2014) Contribution of endogenous glucagon-like peptide 1 to glucose metabolism after Roux-en-Y gastric bypass. Diabetes 63:483-493. https://doi.org/10.2337/ db13-0954

14. Censi S, Mian C, Betterle C (2018) Insulin autoimmune syndrome: from diagnosis to clinical management. Ann Transl Med 6(17):335. https:// doi.org/10.21037/atm.2018.07.32

15. Saxon DR, McDermottMT, Michels AW (2016) Novel management of insulin autoimmune syndrome with rituximab and continuous glucose monitoring. J Clin Endocrinol Metab 101:1931-1934. https://doi.org/10.1210/jc.2016-1097

16. Bodnar TW, Acevedo MJ, Pietropaolo M (2014) Management of non-islet-cell tumor hypoglycemia: a clinical review. J Clin Endocrinol Metab 99:713-722. https://doi.org/10.1210/jc.20133382

Hinweis des Verlags. Der Verlag bleibt in Hinblick auf geografische Zuordnungen und Gebietsbezeichnungen in veröffentlichten Karten und Institutsadressen neutral.

\section{PflegeDossier Cannabinoide}

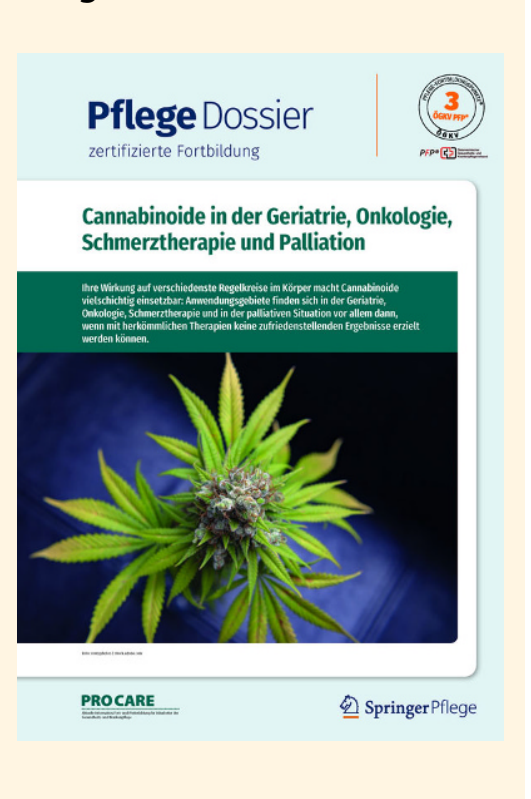

Cannabinoide wirken neuroprotektiv, schmerzstillend und entzündungshemmend und sie zeigen auch verhaltensbezogene positive Effekte. Ein äußerst vielseitiges Cannabinoid ist Dronabinol, das seit 2004 in Österreich als Rezepturarzneimittel verfügbar ist. Seine Einsatzmöglichkeiten sind daher vielschichtig, wobei Dronabinol in der Geriatrie, Onkologie, Schmerzbehandlung und Palliativbetreuung gerne als zusätzliche Therapiemaßnahme genützt wird. Durch ihre große Nähe zum Patienten kommt Diplomierten Gesundheits- und Krankenpflegepersonen auch in diesem Zusammenhang eine wichtige Rolle zu.

Zur Fortbildung für die Gesundheits- und Krankenpflege zum Thema Cannabinoide steht ab sofort ein PflegeDossier von PROCARE/SpringerPflege zur Verfügung, das bei Springer Wien bestellt werden kann: procare@springer.at

\section{Aus dem Internet herunterladen}

Die Fortbildung ist auch online unter www.springermedizin.at/pflegedossier downloadbar, sie ist vom Österreichischen Gesundheits- und Krankenpflegeverband (ÖGKV) zertifiziert und mit 3 Pflegefortbildungspunkten - PFP ${ }^{\circledR}$ bewertet. Die Teilnahme ist durch Unterstützung der Firma Spectrum Therapeutics Austria GmbH kostenlos. 